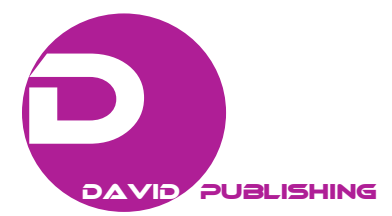

\title{
Fundamental Contact Dynamics Model of a Crawler-Type Vehicle Simulation
}

\author{
Kosuke Sasahara \\ Mechanical CAD Unit, Polytechnic University, Tokyo 187-0035, Japan
}

\begin{abstract}
In this paper, I established a single crawler model with a dynamic contact force considered. I modeled each crawler track using dynamic contact model. By modeling each crawler track, I hope to be able to analyze trafficability of a crawler type vehicle in various road surfaces. First, I think that I established a single crawler model. I modeled a single crawler using Hunt-Crossley model and Coulomb model that handle a nonlinear contact force model. And also, I built a prototype single crawler for comparing the experimental examination with the simulation examination. In advance, the running over limit of the prototype single crawler was analyzed geometrically, and I bore out that it could run over to $163 \mathrm{~mm}$. Therefore, I conducted the experiment examination with step height of $160 \mathrm{~mm}$ and $165 \mathrm{~mm}$ and obtained trajectory data that were allowed to run over and were not allowed to run over. From the result of both the experimental examination and the simulation examination, I bore out the validity of our single crawler model. I described about our single crawler model, the simulation examination and the experimental examination in this paper.
\end{abstract}

Key words: Crawler-type rover, dynamics, Hunt-Crossley.

\section{Introduction}

In recent years, mobile robots are being needed for circumstances in which human presence is not possible such as planetary exploration and/or where high dangers are present like disaster relief missions.

Particularly, in rescue operations it is important to understand the performance of different types of locomotion namely wheel-type, leg-type and crawler-type over the rubble of damaged structures.

Crawler-type robots have advantages over other locomotion methods such as the capability to run on many types of surfaces like soft, loose sand, deep mud and/or soft snow powder and overcoming obstacles that otherwise could get jammed between wheels and small sized holes. On the other side, it has disadvantages like its weight, and that it can cause damage to the road when turning. One important study of the performance of crawler-type robots has been done by Bekker et al. [1], where the

Corresponding author: Kosuke Sasahara, Ph.D., assistant professor, research fields: mechanical engineering, math, machine learning. E-mail: k-sasahara@uitec.ac.jp. terramechanics-based equations that describe the interaction between the ground pressure and the sinkage for wheeled vehicles were presented in 1960.

Later, these terramechanics-based equations were expanded to crawler-type vehicles with the tracks assumed as a board by Wong et al. In 1988, Muro et al. [2] built a model for a crawler vehicle that calculates several forces of the terramechanics-based equations between the ground pressure and the sinkage to design construction and agricultural/forestry machinery cars. Also Abe et al. [3-6] built a model for crawler-type vehicle that presents an optimum design.

This model could express the dynamic characteristics between each track and run over road surfaces.

On the other hand, the surface at a disaster area is considered as the trafficability of uneven surface and different rubble materials. From here onwards, I built crawler model and considered the friction and the contact forces relationship between the tracks and the contact surface.

The aim of this study is to model each track considering the friction and the contact forces when a crawler-type vehicle moves over a disaster area. In 
order to model each track of a crawler-type vehicle, it is necessary to consider a model in which each track contacts an uneven surface. When considering a contact force model, it is conceivable to use Hertz model, Spring-Dashpot model, or Hunt and Crossley model $[7,8]$. The Hunt-Crossley model is a model proposed by Hunt and Crossley in 1975 and is a non-linear contact force model.

In this study, I built a single crawler to verify the contact dynamics model. The track of this prototype single crawler uses a rubber material to make contact with the ground. For this reason, the author assumed that the contact dynamics model was non-linear and adopted a model that fused Hunt-Crossley model and Coulomb model.

In order to verify the proposed model, I performed the experimental examination of one step. Before I tried the experimental examination of one step, the limit step height of one step that the prototype single crawler can run over was analyzed geometrically. From this analysis, I bore out that the prototype single crawler could run over $163 \mathrm{~mm}$ step height. Therefore, the experimental examination was performed when the height of one step was $160 \mathrm{~mm}$ or $165 \mathrm{~mm}$. In this experimental examination, the trajectory data of one step run over are captured motion of a single crawler by the stereo labeling camera.

Then, I compared the trajectory data of one step run over of this experimental examination and of the numerical simulation and bore out the validity of the proposed model.

Therefore, the model proposed in this paper could serve as a basis for future research of crawler-type vehicle over non-flat, nonuniform surfaces.

This paper describes the proposed model, experimental examination, and simulation examination. The rest of this paper is organized as follows. Section 2 describes that I built a single crawler model. Section 3 introduces a prototype single crawler and experimental examination of one step. Section 4 shows the simulation result of a single crawler model. Section 5 is conclusions.

\section{Single Crawler Model Considering the Friction and Contact Force}

I modeled a single crawler that a single crawler is the system of monozoic rigid body, and each crawler track is the reference point of contact (Fig. 1).

Each crawler track of a single crawler moves elliptic orbit clockwise in constant angular velocity. A single crawler obtains the power of moving forward in this motion of crawler track. Since, each reference point turns around clockwise by constant angular velocity of our single crawler model. ${ }^{O} \mathbf{r}_{i}$ and ${ }^{O} \mathbf{V}_{i}$ are the position vector and velocity vector from the system of inertia coordinate in Fig. 1. They are presented in the following equation.

$$
\begin{gathered}
{ }^{o} \mathbf{r}_{i}=\mathbf{r}_{0}+\mathbf{A}^{g} \mathbf{r}_{i} \\
{ }^{o} \mathbf{V}_{i}=\mathbf{v}_{0}+\mathbf{A}^{g} \mathbf{v}_{i}+\omega_{0}{ }^{o} \mathbf{r}_{i}
\end{gathered}
$$

where $\mathbf{r}_{0}$ and $\mathbf{v}_{0}$ represent the position and velocity vector of gravity center of a single crawler from the system of inertia coordinate. $\omega_{0}$ is the constant angular vector of gravity center of a single crawler from the system of inertia coordinate. And also, $\mathbf{A}$ is a coordinate transform matrix, subscript $i$ is presented numbers of each reference point.

Next, I talk about each crawler track. The model of each track is applied that normal direction is elastic deformation model and tangential direction is Coulomb model. First, I talk about the normal direction force model of each crawler track.

Fig. 2 represents that one crawler track is transformed by a contact surface. Then, one crawler track is received the contact force to the normal direction. The contact force $F_{n}$ is the following equation using deformation volume $\delta$.

$$
F(\dot{\delta}, \delta)=b \cdot G(\delta) \cdot \dot{\delta^{q}}+k \cdot \delta^{n}
$$

$b, G, k, q, n$ are the coefficient and scaling exponent that determine the contact force model in Eq. (3).

Eq. (3) is the general expression of contact force model. 


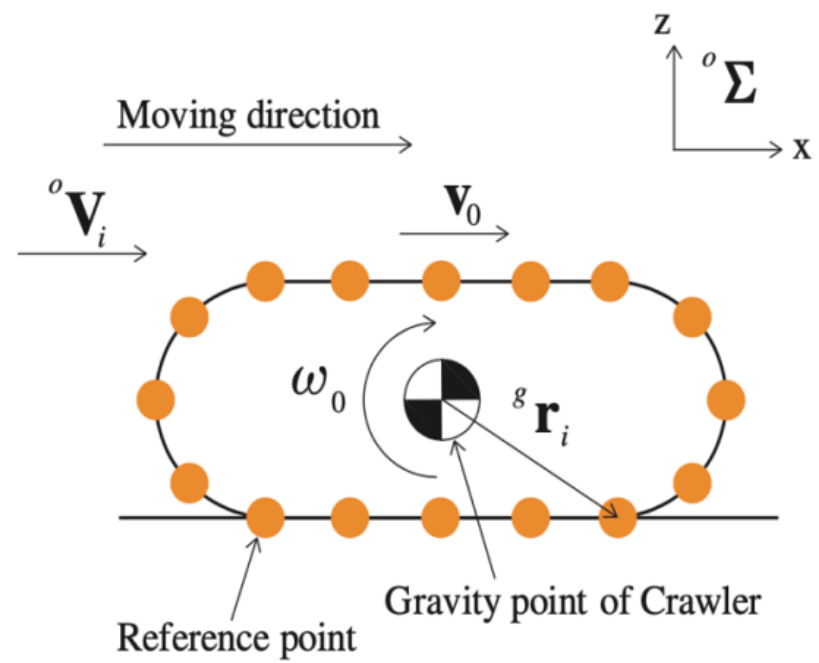

Fig. 1 The image of a single crawler model.

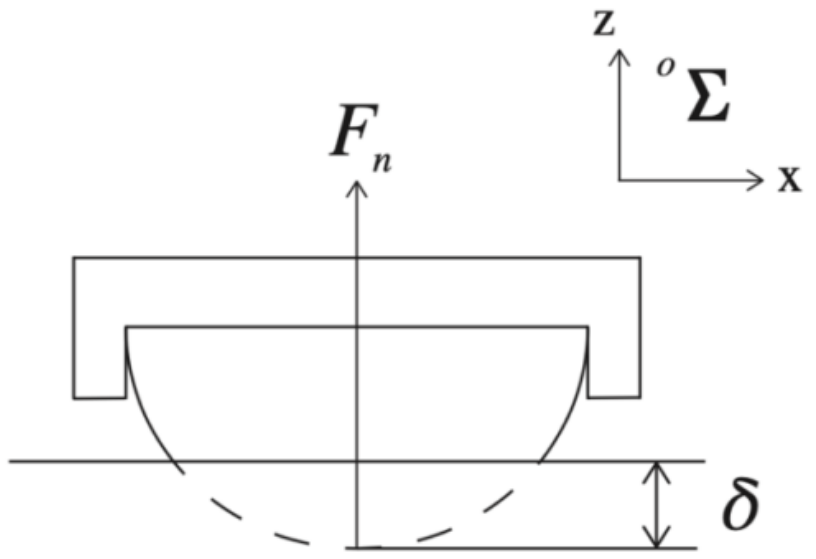

Fig. 2 The normal direction model of crawler track.

I use that the parameter $G$ of Eq. (3) is $\delta^{p} \cdot p$ is also the scaling exponent that determined the contact force model. Then, Eq. (3) is the following equation.

$$
F(\dot{\delta}, \delta)=b \cdot \delta^{p} \cdot \dot{\delta^{q}}+k \cdot \delta^{n}
$$

Eq. (4) is called Hunt-Crossley model. Hunt-Crossley model allows covering nonlinear spring by using $\delta^{p}$. Since, Hunt-Crossley model is the suitable my single crawler model. Eq. (4) applied the normal direction force $f n_{i}$ of each reference point. Eq. (4) is the following equation.

$$
f n_{i}=b \cdot \delta^{p} \cdot{ }^{o} \dot{\boldsymbol{V}}_{i}\left(n_{i}\right)+k \cdot \delta^{n}
$$

where, ${ }^{O} \dot{\boldsymbol{V}}_{i}\left(n_{i}\right)$ is the velocity vector in the normal direction calculated from Eq. (2).

From Eq. (5), the normal direction force of each reference point is calculated.
Next, I talk about the tangential direction force of each crawler track. The tangential direction force model of each crawler track needs to divide up the friction condition that the reference point is the friction of contact surface whether the range of static friction or dynamic friction. This means the physical body changes from static friction range to dynamic friction range by moving itself. By above reason, when each crawler track is the static friction range, I use Hunt-Crossley model and when each crawler track is the dynamic friction range, I use Coulomb model. Since, the tangential force of each crawler track $f t_{i}$ is obtained by the following equation.

$$
\begin{array}{r}
f t_{i}=b \cdot \delta^{p} \cdot{ }^{o} \dot{V}_{i}\left(n_{i}\right)+k \cdot \delta^{n}\left(f t_{i} \leq \mu_{s} \cdot f n_{i}\right)(6) \\
f t_{i}=\mu_{d} \cdot f n_{i}\left(f t_{i}>\mu_{s} \cdot f n_{i}\right)
\end{array}
$$


where, $\delta$ is tangential direction deformation volume of crawler track.

In fact, this deformation is modeled as the difference from initial contact position to present contact position of crawler track. And also, $\mu_{s}$ shows the coefficient of max static friction, and $\mu_{d}$ shows the coefficient of dynamic friction.

The tangential direction force $f t_{i}$ of each reference point, first, is the range of static friction. Then, it is calculated by Eq. (6). After that, when the value of $f t_{i}$ exceeds the value of $\mu_{s} \cdot f n_{i}$, it is calculated by Eq. (7).

Since, the normal direction and tangential force $\mathbf{f}_{i}$ of each crawler is obtained by the following equation.

$$
\mathbf{f}_{i}=\left[\begin{array}{l}
f n_{i} \\
f t_{i}
\end{array}\right]
$$

The torque $\mathbf{t}_{i}$ of each reference is calculated by the following equation using Eq. (8).

$$
\mathbf{t}_{i}={ }^{g} \mathbf{r}_{i} \times \mathbf{f}_{i}
$$

Using Eqs. (8) and (9), the force $\mathbf{F}_{0}$ and torque $\mathbf{T}_{0}$ of gravity center of a single crawler are calculated by the following equations.

$$
\begin{aligned}
\mathbf{F}_{0} & =\sum_{i=1}^{n} \mathbf{f}_{i} \\
\mathbf{T}_{0} & =\sum_{i=1}^{n} \mathbf{t}_{i}
\end{aligned}
$$

Eqs. (10) and (11) are the summation of force $\mathbf{f}_{i}$ and torque $\mathbf{t}_{i}$ from each reference point.

Fig. 3 is the image that the force $\mathbf{F}_{0}$ and torque $\mathbf{T}_{0}$ of single crawler gravity center are calculated from the force $\mathbf{f}_{i}$ and torque $\mathbf{t}_{i}$ of each crawler track.

The single crawler position and velocity of next step are calculated using the force $\mathbf{F}_{0}$ and $\mathbf{T}_{0}$ of single crawler gravity center and the following motion equation.

$$
\mathbf{H}\left[\begin{array}{c}
\dot{\mathbf{v}}_{0} \\
\dot{\omega}_{0}
\end{array}\right]+\mathbf{C}=\left[\begin{array}{c}
\mathbf{F}_{0} \\
\mathbf{T}_{0}
\end{array}\right]
$$

where, $\mathbf{H}$ is inertial term of whole single crawler and C is nonlinear velocity term and gravity term.

At last, I talk about the simulation flowchart. Fig. 4 is the simulation flowchart. In this flowchart, at first, I input initial position and angular velocity of each reference point and the weight and length of single crawler. Using them, I calculate the position ${ }^{0} \mathbf{r}_{i}$ and velocity ${ }^{O} \mathbf{V}_{0}$ of each reference point. Then, the force $\mathbf{f}_{i}$ of each reference point is calculated from the position ${ }^{O} \mathbf{r}_{i}$ and velocity ${ }^{O} \mathbf{V}_{0}$. And the torque $\mathbf{t}_{i}$ of each reference point is calculated from the force $\mathbf{f}_{i}$ of each reference point. The force $\mathbf{F}_{0}$ and the torque $\mathbf{T}_{0}$ of a single crawler gravity center are calculated by summing up the force $\mathbf{f}_{i}$ and the torque $\mathbf{t}_{i}$ of each reference point. The single crawler position and velocity of next step are calculated using the force $\mathbf{F}_{0}$ and the torque $\mathbf{T}_{0}$ of single crawler gravity center and the following motion equation. Each reference point position and velocity update is using the position and velocity of a single crawler. I go back to the first step. A single crawler running trajectory is calculated in each time by calculating iteration.

Z

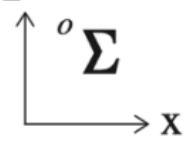

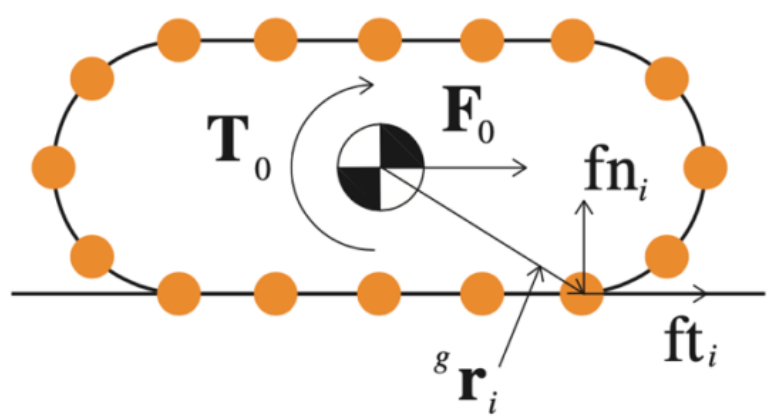

Fig. 3 The image to calculate force and torque of crawler gravity center. 


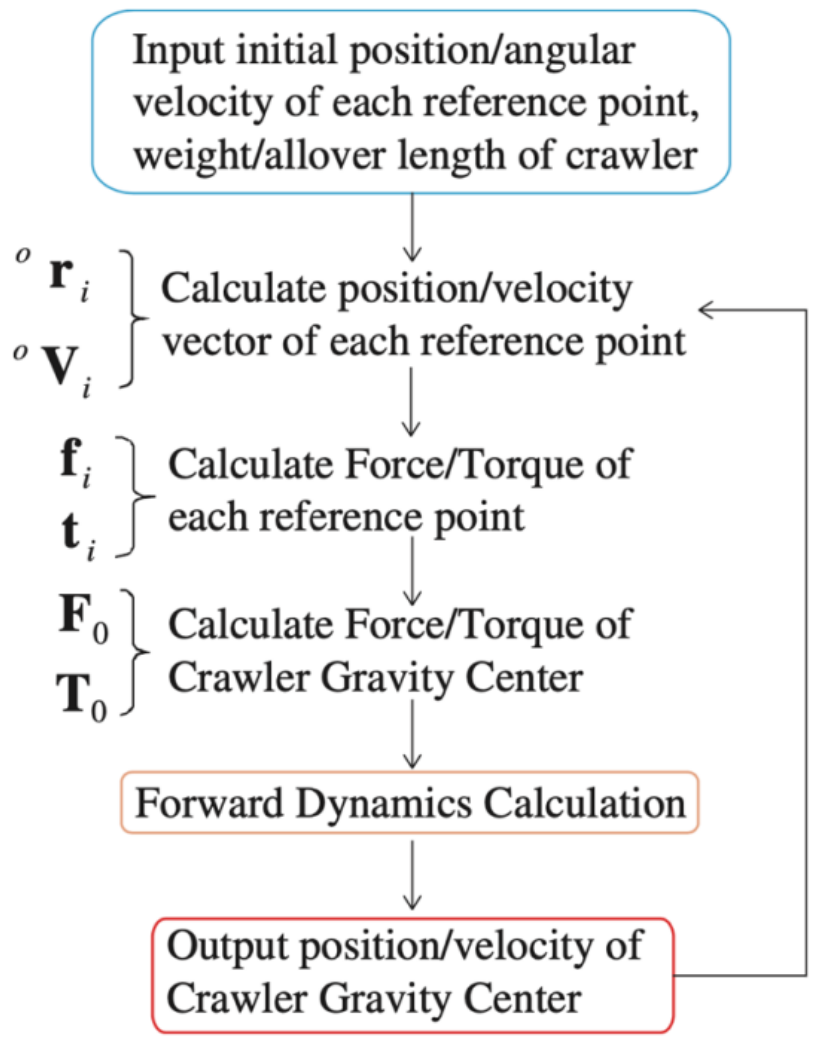

Fig. 4 The flowchart of a single crawler model simulation.

The next section presents that I built a prototype single crawler and the experimental examination.

\section{A Prototype Single Crawler and Experimental Examination of One Step}

At the first, I show I built a single crawler. Fig. 5 is overall view of a single crawler. Fig. 6 is the part of power transmission.

The motor was put in a single crawler inside because I thought an only single crawler is allowed to run. The single crawler traveling direction is to the left side of Fig. 5.

The part of power transmission of Fig. 6 is front of a single crawler. And also, a single crawler spec is shown in Table 1.

Next, I talk about the experimental examination of one step run over. Fig. 7 presented the image of experimental examination of one step run over. The trajectory data of one step run over captured motion of the reflective marker attached front/rear sprocket and gravity center of a single crawler by the stereo labeling camera.

Before I did experimental examination, I established a geometry model because I know a single crawler run over limit step height. Fig. 8 is geometry model.

In Fig. $8, x, L, H, b$ are obtained by the following equations.

$$
\begin{aligned}
& x=\frac{a}{\sin \theta} \\
& L=t+\frac{a}{\sin \theta} \\
& H=L \sin \theta \\
& b=\frac{a}{\cos \theta}
\end{aligned}
$$

Using Eqs. (13)-(16), the limitation step height $h$ of a single crawler running over is the following equation.

$$
h=L \sin \theta+r-\frac{a}{\cos \theta}
$$




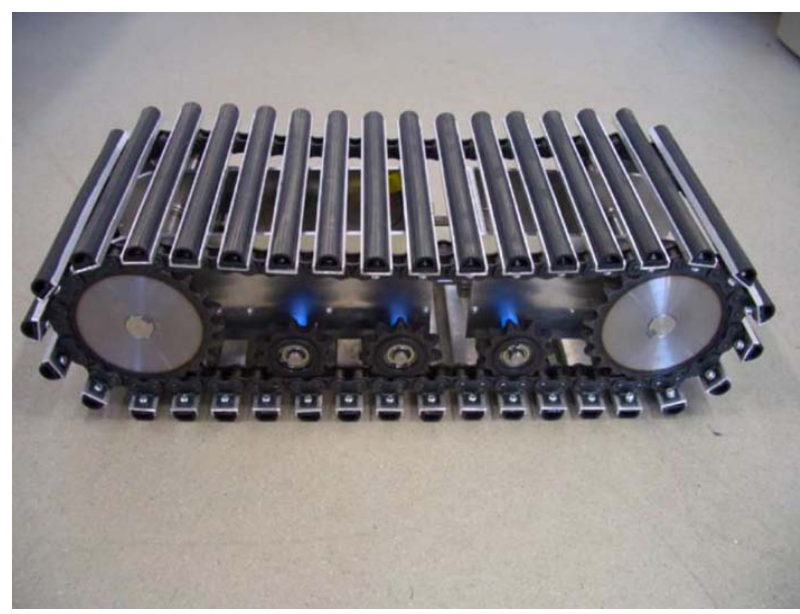

Fig. 5 The overall view of a single crawler.

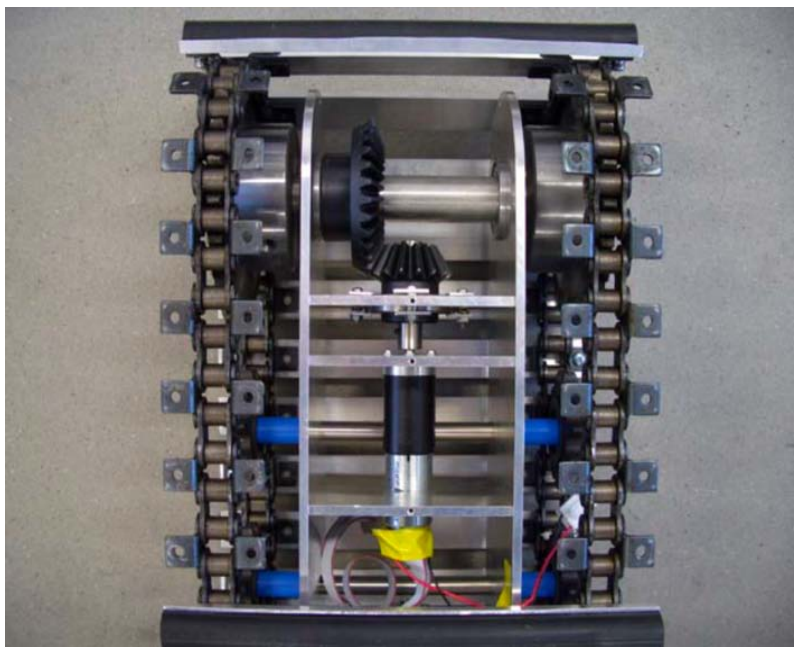

Fig. 6 The part of power transmission.

Table 1 A prototype single crawler spec.

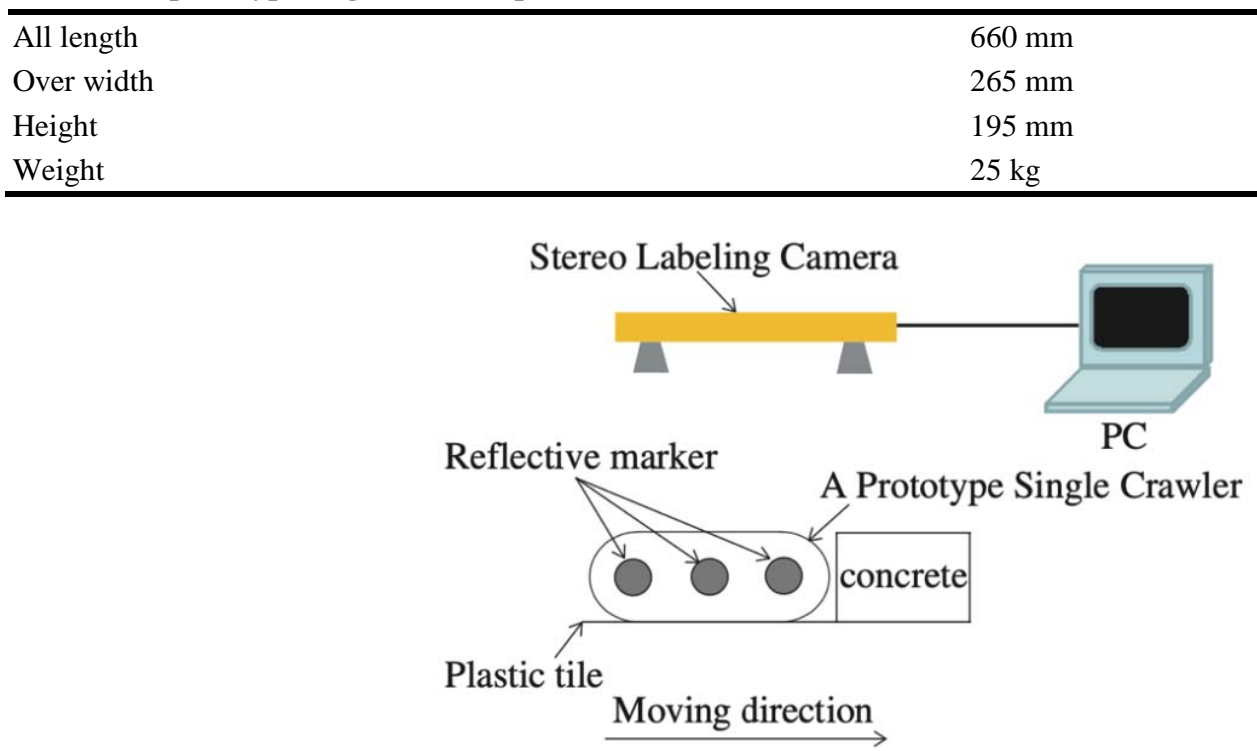

Fig. 7 The image of experimental exam. 


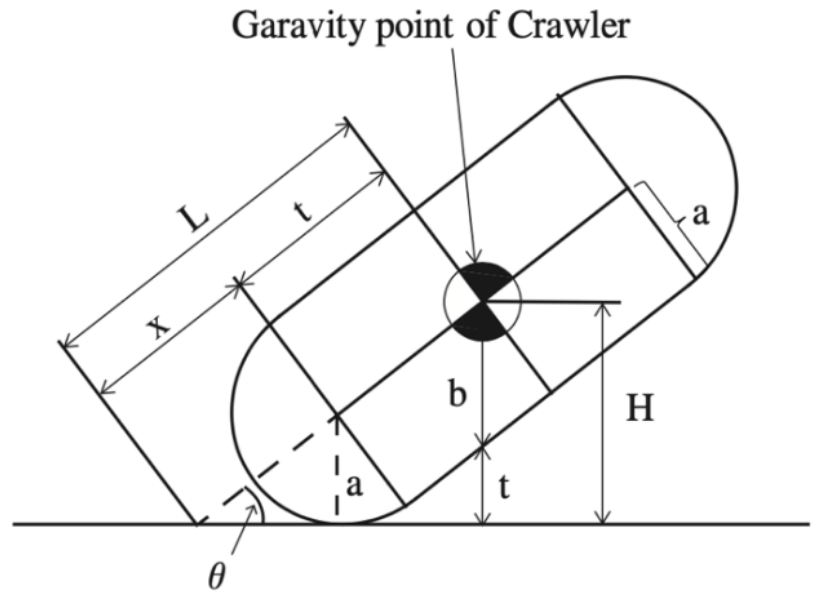

Fig. 8 The geometory model of a single crawler.
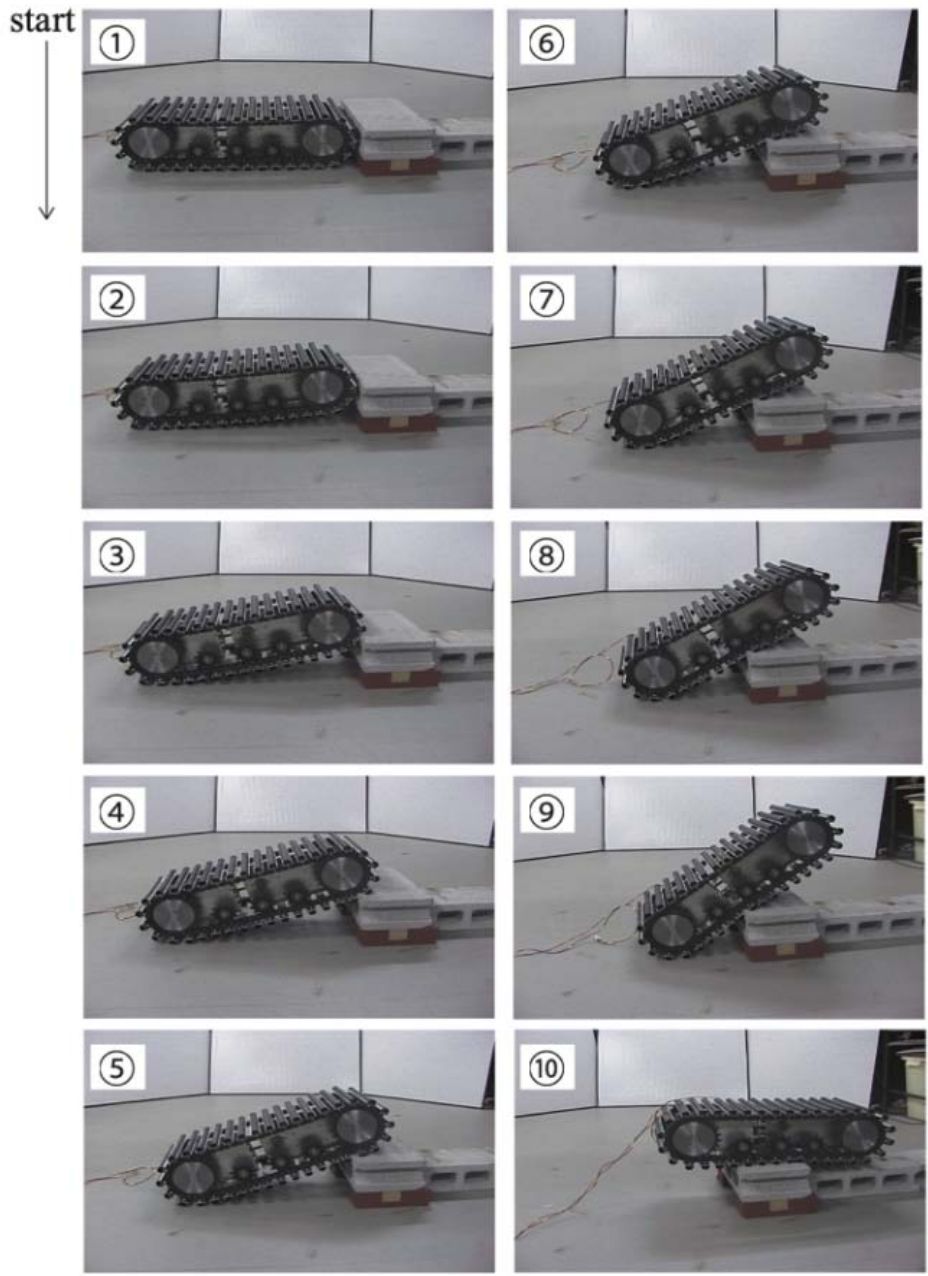

Fig. 9 The appearance image of experimental exam $(160 \mathrm{~mm})$.

I assigned the value $r=1, L=0.25$ of a single crawler parameter. When the tilt angle $\theta$ of a single crawler is about $50^{\circ}$, the limitation step over is about
$163 \mathrm{~mm}$. Then, I tried the step over experimental examination around $165 \mathrm{~mm}$ step height. After, I got the following result in Figs. 9 and 10. 

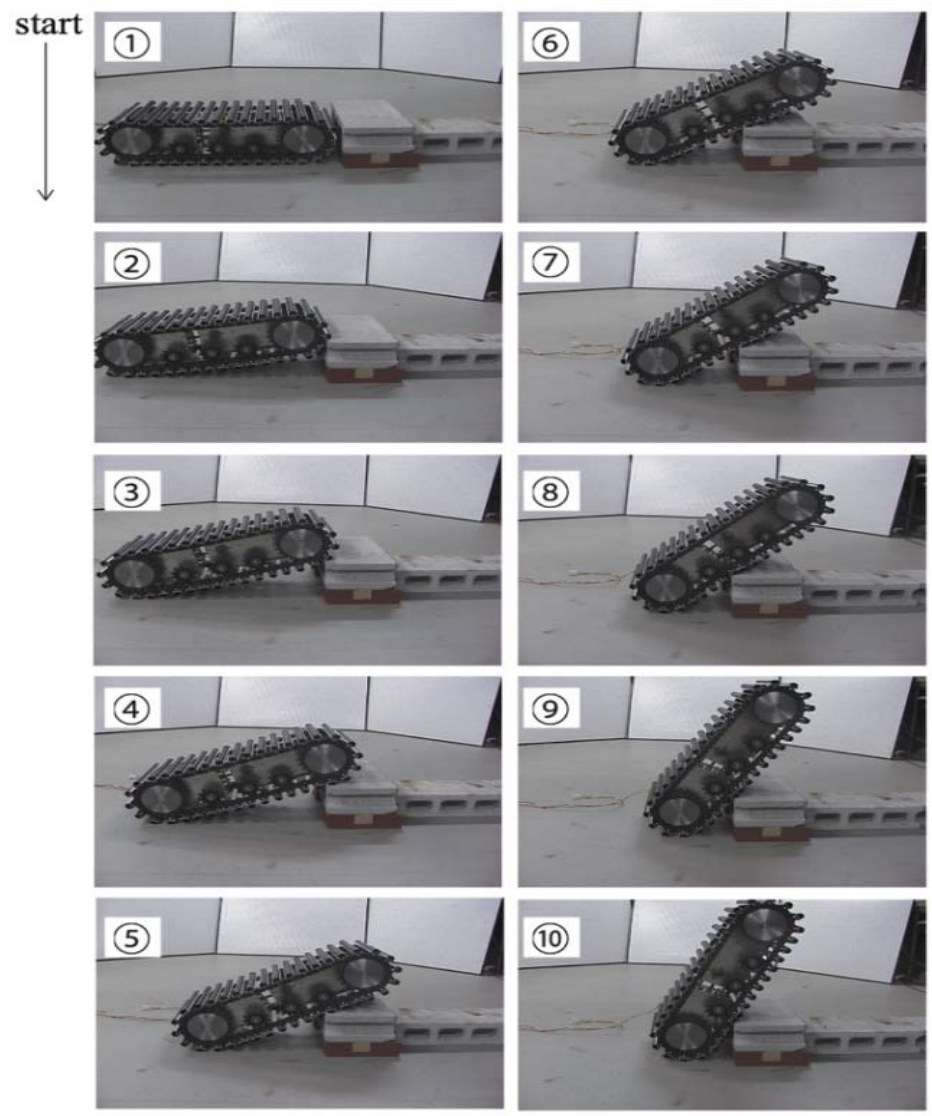

Fig. 10 The appearance image of experimental exam $(165 \mathrm{~mm})$.

Figs. 9 and 10 present the appearance image of a single crawler step over. Fig. 9 is the step height 160 $\mathrm{mm}$ and a single crawler allowed running over step. Fig. 10 is the step height $165 \mathrm{~mm}$ and a single crawler did not allow running over step. And also, Figs. 11 and 12 presented the trajectory data running over one step experimental examination of a single crawler. Fig. 11 presented the step height is $160 \mathrm{~mm}$. Fig. 12 presented the step height is $165 \mathrm{~mm}$. In the above two cases, the initial position of the single crawler was placed on a plastic tile, and the step on which the crawler traveled was concrete.

From this experimental result, I bore out that a single crawler is allowed to run over $160 \mathrm{~mm}$ step height and is not allowed to run over $165 \mathrm{~mm}$ step height. The trajectory of a front and rear sprocket of the single crawler and the position of the center of gravity in this experiment are shown in Figs. 11 and 12.
In Figs. 11 and 12, the blue line is front sprocket trajectory, the green line is gravity center of crawler trajectory, the red line is rear sprocket trajectory. In each of Figs. 11 and 12, a trajectory on which the single crawler is allowed to run over a step and is not allowed to run over a step is shown.

Next, I describe about a numerical simulation experiment that my single crawler runs over a step.

\section{Simulation Exam of a Single Crawler Model}

First, I talk about the simulation condition. Table 2 presents the parameter of elastic deformation model and the coefficient of contact surface.

The parameter values $k$ and $b$ of elastic deformation model set the value of keeping balance between a single crawler model and normal direction contact force in Eqs. (5) and (6). The parameter values $p$ and $n$ of scaling exponent determined the contact 


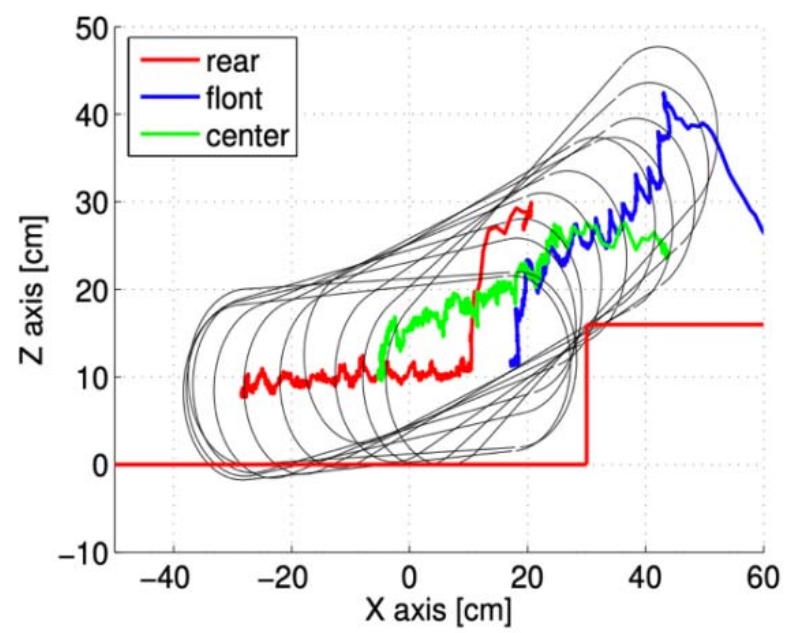

Fig. 11 The trajectory data of experimental exam $(160 \mathrm{~mm})$.

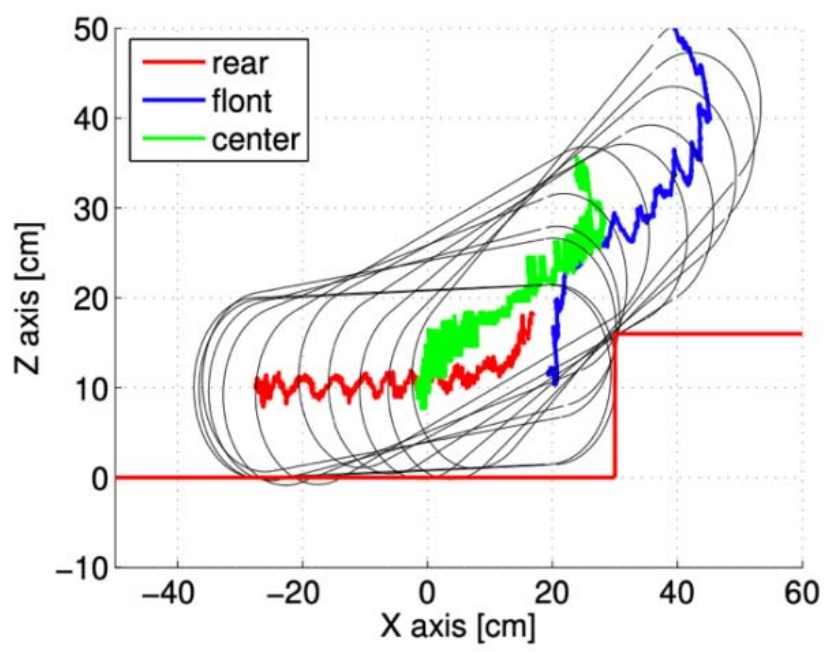

Fig. 12 Output from improved acquisition algorithm.

Table 2 Simulation parameter.

\begin{tabular}{ll}
\hline Elastic deformation model parameter & $k=11,000$ \\
& $b=15,000$ \\
\hline \multirow{2}{*}{ Scaling exponent } & $p=0.5$ \\
& $p=1.5$ (wall) \\
Concrete friction & $n=1.5$ \\
& $\mu_{d}=1.2$ \\
Initial contact surface friction & $\mu_{s}=1.44$ \\
& $\mu_{d}=0.5$ \\
$\mu_{s}=0.6$
\end{tabular}

force model in Eqs. (5) and (6). Here, the above parameters were determined in consideration of Ref. [9] and the deformation of the rubber used for the prototype single crawler.

And also, I estimate the friction coefficient of contact surface by doing experimental examination.
The experimental examination is that a prototype single crawler is pulled by the tangential direction on each surface. The tangential direction force is measured by spring scales. The friction coefficient was obtained by dividing the measurement value by weight of a single crawler. 


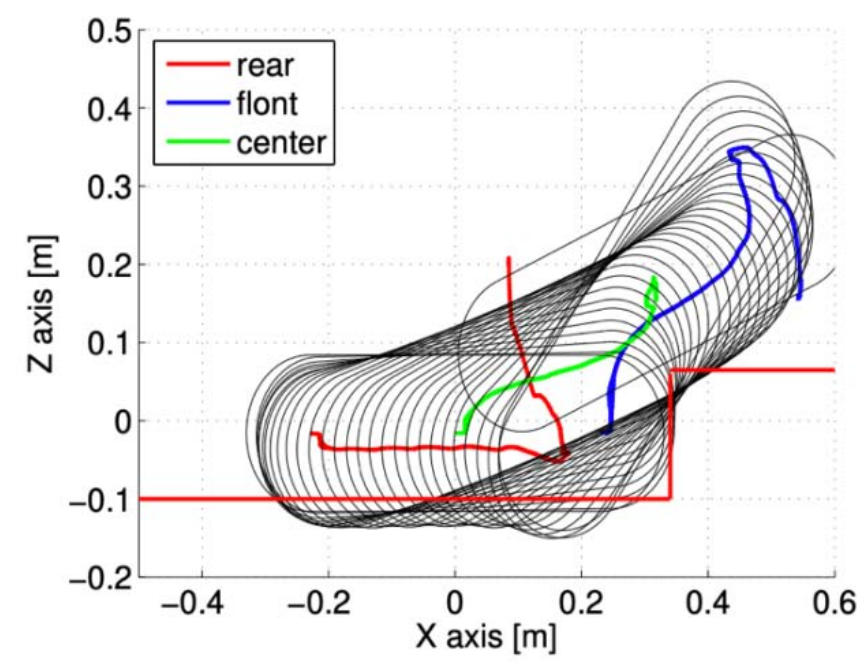

Fig. 13 The trajectory data of simulation exam $(160 \mathrm{~mm})$.

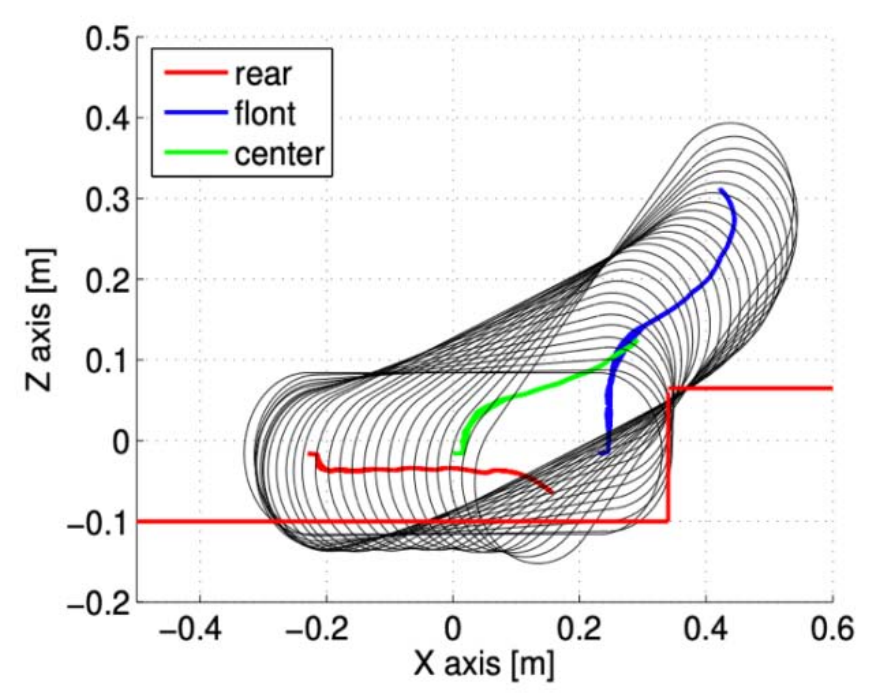

Fig. 14 The trajectory data of simulation exam (165 mm).

I run simulation examination in $160 \mathrm{~mm}, 165 \mathrm{~mm}$, and low friction of tangential direction contact surface. The sampling time of simulation examination is $1 \mathrm{~ms}$.

Figs. 13 and 14 are the simulation examination result. The blue line is front sprocket trajectory, the green line is gravity center trajectory, and the red line is rear sprocket trajectory.

Fig. 13 is $160 \mathrm{~mm}$ of the step height. In this case, front sprocket trajectory moved to step side and rear sprocket lifted up. Since, a single crawler model was allowed to run over $160 \mathrm{~mm}$ step height. On the other hand, in Fig. 14, front sprocket trajectory did not move to step side and rear sprocket did not lift up.
Since, a single crawler model was not allowed to run over $165 \mathrm{~mm}$ step height. This result is the same experimental examination result in Figs. 13 and 14. From this experimental result, I bore out my model validity in this case. And also, I was able to establish a single crawler with the dynamics considered.

\section{Conclusions}

I tried to establish a single crawler model in uneven contact surface. When a single crawler runs over uneven surface, each crawler track contacts different surfaces. Then, I established each crawler track model using the contact mechanics model. Hunt-Crossley 
model and Coulomb model were used for the contact mechanics model. For I bore out the validity of my single crawler model, I run the simulation examination and built a prototype single crawler and did experimental examination.

Before I tried the step over experimental examination, I geometrically analyzed the single crawler running the limitation step over. By this analysis, I bore out that the single crawler could run over to the $163 \mathrm{~mm}$ step height.

Based on this analysis result, I tried the experimental examination with the simulation examination result in 160 mm, 165 mm step height. From the results of this experiment, I bore out that my single crawler was allowed to run over $160 \mathrm{~mm}$ step height and was not allowed to run over $165 \mathrm{~mm}$ step height.

Next, I tried to use a contact mechanics model to reproduce the experiment examination using my single crawler in simulation examination. The result of both experimental examination and simulation examination is about the same trajectory.

And also, I bore out the validity of the contact mechanics model using Hunt-Crossley model and Coulomb model.

Therefore, the model proposed in this paper could be useful as a basis for future research of crawler-type vehicle over non-flat, nonuniform surfaces.

However, this paper does not discuss the parameter estimation of the contact mechanics model in numerical simulation.
As a future work, I will conduct an experiment when changing the friction of surface that the crawler comes into contact with, and an experiment when changing to a complicated road surface.

\section{References}

[1] Bekker, M. G. 1960. Off-The-Road Locomotion. Ann Arbor: The University of Michigan Press.

[2] Muro, T. 1993. Terramechanics-Travel Dynamics. Gihodoshuppan.

[3] Nakagawa, C., Abe, M., and Ito, H. 1993. "Dynamic Interaction between Crawler Shoes and Road." Transactions of the Japan Society of Mechanical Engineers C 59 (560): 1075-9.

[4] Nakagawa, C., Abe, M., and Ito, H. 1993. "Dynamic Interaction between Crawler Shoes and Road." Transactions of the Japan Society of Mechanical Engineers C 59 (563): 2092-7.

[5] Nakagawa, C., Abe, M., and Ito, H. 1998. "Design of a Single Frequency GPS Software Dynamic Interaction between Crawler Shoes and Road." Transactions of the Japan Society of Mechanical Engineers C 64 (617): 307-15.

[6] Nakagawa, C., Abe, M., and Ito, H. 1998. "Dynamic Interaction between Crawler Shoes and Road." Transactions of the Japan Society of Mechanical Engineers C 64 (617): 316-23.

[7] Hunt, K., and Crossley, F. 1975. "Coefficient of Restitution Interpreted as Damping in Vibroimpact." ASME J. Appl. Mech. 42: 440-5.

[8] Gilardi, G., and Sharf, I. 2002. "Literature Survey of Contact Dynamics Modelling." Mechanism and Machine Theory 37: 1213-39.

[9] Kuwabara, G., and Kono, K. 1987. "Restitution Coefficient in a Collision between Two Spheres." Japanese Journal of Applied Phisics 26 (8): 1230-3. 